\title{
Pseudomonas aeruginosa among Cystic Fibrosis patients: epidemiology, risk factors and infection control
}

\author{
Aysegul Karahasan ${ }^{1}$, Gamze Alci ${ }^{1}$, Can Akin², Ayca Cakan ${ }^{2}$, Eren Sarioglu², Batuhan \\ Basci $^{2}$, Yasemin Gokdemir ${ }^{1}$, Ela Eralp ${ }^{1}$, and Bulent Karadag ${ }^{3}$ \\ ${ }^{1}$ Marmara University Training and Research Hospital \\ ${ }^{2}$ Marmara University School of Medicine \\ ${ }^{3}$ Marmara University Faculty of Medicine
}

June 4, 2020

\begin{abstract}
Introduction and Objective: Microbiological surveillance guides the antibiotic therapy that plays an important role in maintaining the cystic fibrosis (CF) patients in stable clinical condition. Materials and Method: Respiratory samples (495 sputum, 711 deep pharyngeal swab) from $253 \mathrm{CF}$ patients aged 0-43 were cultivated in conventional media and cumulative antibiogram was determined. A 24 questioned survey was applied to 119 patients for a 3 months period to measure demographic variabilities and knowledge about infection control. Results: The most common pathogen was Pseudomonas aeruginosa (45.7\%), followed by Staphylococcus aureus (36\%). P. aeruginosa was isolated from $60 \%$ of sputum samples and $28.5 \%$ of deep pharyngeal swab samples. High rate of resistance was detected to ceftazidime, amikacin and ciprofloxacin that are often preferred in treatment. Low monthly income, more than 9 visits to CF clinic in the last year, hospitalisation in the last year, spending more than 3 hours in CF clinic were significantly associated with P. aeruginosa colonisation. Health care providers was the main source of information $(62.2 \%)$.Vast majority of the patients $(88.2 \%)$ knew that pathogens can be transmitted between the patients and infections could be reduced by using a mask, handwashing and by cleaning nebulizers as declared by $87.4 \%$ of participants clean the nebulizer after every usage and $93.3 \%$ of them dry it properly. Conclusion: Prevention of infections in CF patients is possible only if patients and their families are alert and have sufficient information about infection control that will provide great improvements in the prognosis. Key Words: P. aeruginosa, Cystic Fibrosis, Antibiotic Resistance, Infection control

Pseudomonas aeruginosa among Cystic Fibrosis patients: epidemiology, risk factors and infection control

Karahasan Yagci A. ${ }^{1 *}$,Alci $\mathrm{G}^{1}$, Akin $\mathrm{C}^{2}$, Cakan $\mathrm{A}^{2}$, Sarioglu $\mathrm{E}^{2}$, Basci $\mathrm{B}^{2}$, Gökdemir $\mathrm{Y}^{3}$, Erdem Eralp E. ${ }^{3}$, Karadag B. ${ }^{3}$

${ }^{1}$ Medical Microbiology Department, Marmara University Hospital, Istanbul

${ }^{2}$ Marmara University Faculty of Medicine, Istanbul

${ }^{3}$ Pediatric Pulmonology Division, Marmara University Faculty of Medicine, Istanbul

*corresponding author

Gmail:aysegulkarahasan@gmail.com
\end{abstract}

Tel: 905325823582

ABSTRACT 
Introduction and Objective: Microbiological surveillance guides the antibiotic therapy that plays an important role in maintaining the cystic fibrosis $(\mathrm{CF})$ patients in stable clinical condition.

Materials and Method: Respiratory samples (495 sputum, 711 deep pharyngeal swab) from $253 \mathrm{CF}$ patients aged 0-43 were cultivated in conventional media and cumulative antibiogram was determined. A 24 questioned survey was applied to 119 patients for a 3 months period to measure demographic variabilities and knowledge about infection control.

Results: . The most common pathogen was Pseudomonas aeruginosa (45.7\%), followed by Staphylococcus aureus (36\%).P. aeruginosa was isolated from $60 \%$ of sputum samples and $28.5 \%$ of deep pharyngeal swab samples. High rate of resistance was detected to ceftazidime, amikacin and ciprofloxacin that are often preferred in treatment. Low monthly income, more than 9 visits to $\mathrm{CF}$ clinic in the last year, hospitalisation in the last year, spending more than 3 hours in $\mathrm{CF}$ clinic were significantly associated with $P$. aeruginosacolonisation. Health care providers was the main source of information $(62.2 \%)$ ).Vast majority of the patients $(88.2 \%)$ knew that pathogens can be transmitted between the patients and infections could be reduced by using a mask, handwashing and by cleaning nebulizers as declared by $87.4 \%$ of participants clean the nebulizer after every usage and $93.3 \%$ of them dry it properly.

Conclusion: Prevention of infections in CF patients is possible only if patients and their families are alert and have sufficient information about infection control that will provide great improvements in the prognosis.

Key Words: P. aeruginosa, Cystic Fibrosis, Antibiotic Resistance, Infection control

\section{INTRODUCTION}

Cystic fibrosis is the most common chronic respiratory disease among European Caucasian children. Cystic fibrosis incidence in Northern European countries is approximately 1 in 3000 births while the worldwide prevalence varies considerably. It is well known that, the most common pathogen isolated from the respiratory tract of patients with cystic fibrosis is $P$. aeruginosa. Once placed in the host, $P$. aeruginosa is usually permanent and undergoes some changes such as mucoid colony morphology, loss of movement, hypo-expression of various exotoxins and other products ${ }^{1,2}$. P. aeruginosa prevalence is $20 \%$ in patients younger than 5 years of age and increases to $70 \%$ at 18 years of age ${ }^{3}$. Pulmonary functions regress more rapidly as a result of chronic lung infection with $P$. aeruginosa and caused early death of CF patients. Antimicrobial treatment play one of the most important roles in the survival of CF patients. $P$. aeruginosa isolated in the early stages of the disease is usually susceptible to antibiotics, but progresses growing antibiotic resistance due to antibiotic pressure in time ${ }^{4}$. Awareness of patients about infection control is vital for prevention of resistant bacterial colonization. Continuous surveillance of patients and informative educational activities are the most important facilities to increase the quality of patient's life.

In this study, we retrospectively analysed the incidence of bacterial pathogens among respiratory samples in our CF patients to determine the role of $P$. aeruginosa and evaluated antimicrobial resistance by facilityspecific antibiogram. We also performed a survey to analyse how well patients and families understand the principles of infection control and what are the knowledge, attitudes, and practices of CF patients and their families.

\section{MATERIAL AND METHOD}

1. Two hundred fifty eight CF patient's sputum or deep pharyngeal swab (DPS) samples were referred to our center in 2018. Bacterial isolation was done using conventional culture media and colonies were identified by MALDI-TOF (Biomerioux, France) method. Antimicrobial susceptibility test was performed according to EUCAST criteria. Cumulative antibiogram data obtained according to CLSI M39 A4 document.

2. A survey has been conducted between November 2018- January 2019. The Marmara University Medical Faculty Research Ethics Committee approved the study. Written and oral consents were obtained from the parents of the patients. We used 24 questioned survey to investigate demographic properties and 
knowledge about infection control in CF patients or their caregivers. SPSS 24.0 was used to analyse data, chi square test was performed as significance measurement $(\mathrm{p}<0,05)$.

3. Selected multidrug resistant strains (n: 67) isolated from the patients during our survey were genotyped by AP-PCR ${ }^{5}$. DNA was extracted by using the DNA purification kit (GENMARK) and ERIC-1 and ERIC-2 primers were used. PCR products were visualized in agarose 1, 5\% gels stained with ethidium bromide.

\section{RESULTS}

We evaluated respiratory samples of 253 CF patients for a one year period, January- December 2018. Median age of the patients was 17 with a range of 0-43. Among these patients, 114 (45.1\%) were female. Microorganisms isolated from respiratory samples (495 sputum, 711 deep pharyngeal swab) were given in Table 1 . The most common pathogen wasPseudomonas aeruginosa (45.7\%), followed by Staphylococcus aureus (36\%). $P$. aeruginosa was isolated from $60 \%$ sputum samples and $28.5 \%$ deep pharyngeal swab samples. Single pathogen was isolated in $71 \%$, double pathogens in $26 \%$ and triple pathogens in $3 \%$ of the samples.

The highest colonization rate for was detected in the age groups of 6-15 as shown in Table 2. Median age of the $P$. aeruginosa isolated patients was 17 with a range of $0-43$.

Antimicrobial susceptibility rate of $P$. aeruginosa strains is given in Table 3 and cumulative data is shown in Table 4. High rates of resistance was detected to ceftazidime, aminoglycosides and ciprofloxacin.

Demographic features of 119 patients that a prospective survey was conducted for a 3 months period, November 2018- January 2019 were given in Table 5. Median age was 10.49 and 19 of them were older than 18 years age. Laboratory data revealed that 70 (58.8\%) of them were colonised by Pseudomonas aeruginosa . Low monthly income, more than 9 visits to CF clinic in the last year, hospitalisation in the last year, spending more than 3 hours in $\mathrm{CF}$ clinic were significantly associated with $P$. aeruginosa colonisation. Our patients and their families mainly have had their knowledge about $\mathrm{CF}$ from health care providers $(62.2 \%)$.

As seen in Table 6 , vast majority of the patients (88.2\%) knew that pathogens can be transmitted between the patients and infections could be reduced by using a mask, handwashing and by nebulizers. $87.4 \%$ of the participants declare that they clean the nebulizer after every usage and $93.3 \%$ dry it properly.

Despite the fact that health care providers give training on using a nebulizer, $35.3 \%$ of the patients do not have a spare nebulizer. Seventy six percent of respondents agreed that CF patients should be at least 2 metres apart and should avoid close contact with others with CF in the clinic. Comparison of isolated strains with molecular methods is the best way to evaluate transmission of the strains. In our study, we have genotyped only limited number of strains by ERIC-PCR and our preliminary data shows no cross colonisation of $P$. aeruginosa strains between patients.

\section{DISCUSSION}

Significant advances in therapies and outcomes for people with CF over the past 30 years have brought hope and optimism to clinicians, researchers, and most importantly, individuals and families with $\mathrm{CF}^{6}$. However, although life expectancy for people with cystic fibrosis has increased substantially, the disease continues to limit the quality of life for the patients.

Chronic respiratory infections play an important role in morbidity and mortality in CF. $S$ aureus is often the first respiratory pathogen identified in respiratory secretions in young children with CF although there is some controversy as to whether they should receive antibiotic prophylaxis to treat an $S$. aureus infection 7.P. aeruginosa is the most common pathogen encountered in adults and is related promoting an accelerated decline of pulmonary function. Importantly, $P$. aeruginosa exhibits significant resistance to innate immune effectors and to antibiotics, in part, by expressing specific virulence factors (e.g., antioxidants and exopolysaccharides) and by acquiring adaptive mutations during chronic infection ${ }^{2}$. We analysed 1151 respiratory samples of CF patients (n: 253) and revealed that the most common pathogen wasPseudomonas aeruginosa 
(45.7\%), followed by Staphylococcus aureus (36\%). P. aeruginosa was isolated from $60 \%$ sputum samples and $28.5 \%$ deep pharyngeal swab samples. Median age of the P. aeruginosa isolated patients was 17 .

An essential component in the management of CF respiratory disease is the elimination of $P$. aeruginosa through the use of anti-pseudomonal antibiotic therapy ${ }^{8}$. Active antibiotic therapy can reduce the deterioration of lung function associated with chronic infection and results in positive implications for the long-term prognosis in CF. Delayed onset of chronic $P$. aeruginosa infection and better pulmonary function may reduce treatment burden and morbidity and improve quality of life and overall life expectancy ${ }^{9}$.

The use of inhaled antibiotics, such as tobramycin, and aztreonam, as chronic suppressive therapy is a mainstay of $\mathrm{CF}$ respiratory infection management by aiming . higher airway drug concentrations . However, recurrent and prolonged systemic or inhaled antibiotic therapy has been directly linked with the emergence of resistance ${ }^{10}$. The use of ceftazidime monotherapy has been found to be related with outbreaks of multidrugresistant $\mathrm{P}$. aeruginosa infections and, it is recommended that two antibiotics, usually a combination of aminoglycoside and a beta-lactam agent, be administered to treat CF patients with sensitive $P$. aeruginosa infection to prevent spread of infection ${ }^{11}$. Unfortunately, nearly half of our isolates were resistant to these widely used antimicrobials indicating in a near future it would be even more difficult to eradicate $P$. aeruginosa from the patients. Given the higher pathogenicity of resistant $P$. aeruginosa, it is preferable to isolate patients infected with these strains in individual hospital wards to reduce patient to- patient transmission, and to decrease the spread of resistant strains of P. aeruginosa among patients with CF. We also follow this approach in our clinics .

Although direct and indirect contact with infectious secretions and droplets are known transmission mechanisms for CF pathogens, the role of airborne transmission via droplet nuclei has been less certain. Recovery of CF pathogens from the air has been variably demonstrated under different conditions. Zuckerman et al reported that $8 \%$ of air samples obtained 3 feet from participants in CF clinics contained their respiratory tract pathogens ${ }^{12}$. CF infection control guidelines published in 2003 sought to minimise potential contact and droplet transmission of pathogens and recommended cohort segregation according to microbiological status, single room inpatient accommodation and a separation distance of 1-meter between people with CF. The updated CF infection control guidelines published in 2014 recommended a separation distance of 2-meters and included the specific recommendation for people with $\mathrm{CF}$ to wear a surgical mask in communal areas of healthcare facilities ${ }^{13}$. Only $75.6 \%$ of our patients declared that "I pay attention to the fact that there should be approximately 2 meters of distance between my CF relative and the other patients". In order to reduce the risk of transmission of pathogens transmitted by the respiratory system, informative studies should be conducted on this subject. Particular attention should be paid to the presence of colonized patients. As a result, measures should be taken to reduce the risk of cross-over to improve the quality of life and prevent deterioration of CF patients. Patients and their relatives should be informed about this issue. The implementation of these practices in the outpatient clinic should be closely monitored.

In our study $85.7 \%$ of survey participants knew that use of masks in hospital reduces the risk of transmission among the patients. Accumulated evidence led to updated recommendations that patients with CF should wear masks in common areas of healthcare facilities to prevent acquisition of pathogens by droplets from infected patients with CF. Mask use by patients with CF is not advised in exam rooms but is recommended in common areas such as waiting rooms and clinic corridors. Wood et al demonstrated that surgical and N95 masks both significantly reduce the potential for bio aerosol dispersal with $>90 \%$ reduction in the mean total CFUs. Face masks reduce cough generated $P$. aeruginosa aerosols, with the surgical mask providing enhanced comfort ${ }^{14}$.

Awareness of patients is vital for infection control. In our patients, health professionals played a key role in patient awareness. In addition, the guiding effect of non-governmental organizations was striking. A significant majority of the patients stated that they received information from non-governmental organizations such as KIFDER (Cystic Fibrosis Family Association). Therefore, conducting joint studies with such organizations may facilitate access to CF patients. In a large scale survey, Miroballi et al assessed infection control knowledge, attitudes, and practices among CF patients and their families at 17 randomly selected 
CF centers and stated that $65 \%$ of respondents were aware of the CF infection control guidelines, but only $30 \%$ had discussed them more than once with their CF care team ${ }^{15}$. Patients and families with more frequent discussions with their $\mathrm{CF}$ care team had increased knowledge of infection control principles and of pathogen transmission, increased confidence in their ability to perform certain infection control practices, and increased perception of the health benefits of the guidelines.

Studies have demonstrated that unrelated people with CF can harbor genotypically indistinguishable strains, suggesting person-to-person spread of $P$. aeruginosa ${ }^{16 .}$. In a large study of Australian CF clinics, presence of six major clonal groups shared by multiple patients were detected by ERIC- PCR with the dominant clonal group infecting $38 \%$ of all patients ${ }^{17}$. We also genotyped limited number of strains by ERIC- PCR and detected no predominant type. This preliminary data may suggest that suggesting no signs of cross-infection in our outpatient clinic however there is a need of large scale detailed study to confirm this result.

Considering the probability of cross-infection in CF patients, maintaining the importance given to infection control is a critical point in the course of the disease. The length of time spent in various polyclinics in the hospital is a factor that makes it difficult to control infection. More than 9 visits to CF clinic in the last year, hospitalisation in the last year, spending more than 3 hours in CF clinic were significantly associated with $P$. aeruginosacolonisation in our group. . Therefore, measures should be taken to minimize the time that CF patients spend in the hospital. We observed that our patients and their relatives were open to information sharing and learning. In this direction, organizing training seminars and distributing brochures about CF will be an appropriate attitude to reach and raise awareness of patients and their relatives.

\section{REFERENCES}

1. Bhagirath AY, Li Y, Somayajula D, Dadashi M, Badr S, Duan K. Cystic fibrosis lung environment and Pseudomonas aeruginosa infection. BMC Pulm Med. 2016;16(1): 174- 196

2. Malhotra S, Hayes D Jr, Wozniak D. Cystic Fibrosis and Pseudomonas aeruginosa: the Host-Microbe Interface. Clin Microbiol Rev. 2019 May 29;32(3):e00138-18. doi: 10.1128/CMR.00138-18.

3. Ehsan Z, Clancy JP. Management of Pseudomonas aeruginosa infection in cystic fibrosis patients using inhaled antibiotics with a focus on nebulized liposomal amikacin. Future Microbiol 2015;10(12):1901-12. doi: 10.2217/fmb.15.117. Epub 2015 Nov 17.

4. Nixon GM, Armstrong DS, Carzino R, Carlin JB, Olinsky A, Robertson CF, Grimwood K. Clinical outcome after early Pseudomonas aeruginosainfection in cystic fibrosis. J Pediatr 2001; 138: 699-704.

5. Auda IG, Al-Kadmy IM, Kareem SM, Lafta AK, A'Affus MH, Khit IA, Al Kheraif AA, Divakar DD, Ramakrishnaiah R. RAPD- and ERIC-Based Typing of Clinical and Environmental Pseudomonas aeruginosa Isolates. J AOAC Int. 2017 Mar 1;100(2):532-536

6. Sanders DB, Fink AK Background and Epidemiology. .Pediatr Clin North Am. 2016 Aug;63(4):567-84. doi: $10.1016 /$ j.pcl.2016.04.001.

7. Smyth AR. Pseudomonas eradication in cystic fibrosis: who will join the ELITE? Thorax 2010;65(4):281-2.

8. Gibson RL, Burns JL, Ramsey BW. Pathophysiology and management of pulmonary infections in cystic fibrosis. Am J Respir Crit Care Med 2003;168:918-51

9. Sandy Z. P. Lim, Dominic A. Fitzgerald Treating resistant Pseudomonas aeruginosa lung disease in young children with cystic fibrosis. Paediatr Respir Rev 2018; 33-36

10. Bell SC, Mall MA, Gutierrez H, Macek M, Madge S, Davies JC, Burgel PR, Tullis E, Castaños C, Castellani C, et al. The Future of Cystic Fibrosis Care: A Global Perspective Lancet Respir Med. 2020 Jan;8(1):65-124

11. Kidd TJ, Canton R, Ekkelenkamp M, Johansen HK, Gilligan P, LiPuma JJ, Bell SC, Elborn JS, Flume PA, VanDevanter DR, Waters VJ; Antimicrobial Resistance in Cystic Fibrosis International Working Group. Defining Antimicrobial Resistance in Cystic Fibrosis. J Cyst Fibros 2018 Nov;17(6):696-704.

12. Zuckerman JB, Zuaro DE, Prato BS, Ruoff KL, Sawicki RW, Quinton HB, Saiman L. Bacterial contamination of cystic fibrosis clinics. J Cyst Fibros 2009;8:186-19

13. Saiman L, Siegel JD, LiPuma JJ, Brown RF, Bryson EA, Chambers MJ, Downer VS,Fliege J, Hazle 
LA, Jain M, Marshall BC, O'Malley C, Pattee SR, Potter-Bynoe G, Reid S, Robinson KA, Sabadosa KA, Schmidt HJ, Tullis E, Webber J, Weber DJ, Cystic Fibrous F, Society for Healthcare Epidemiology of America. Infection prevention and control guideline for cystic fibrosis: 2013 update. Infection Control and Hospital Epidemiology 2014; 35 Suppl 1: S1-S67.

14. Wood ME, Stockwell RE, Johnson GR, Ramsay KA, Sherrard LJ, Jabbour N, Ballard E, O'Rourke P, Kidd TJ, Wainwright CE, Knibbs LD, Sly PD, Morawska L, Bell SC. Face Masks and Cough Etiquette Reduce the Cough Aerosol Concentration of Pseudomonas aeruginosa in People with Cystic Fibrosis.Am J Respir Crit Care Med. 2018 Feb 1;197(3):348-355.

15. Miroballi Y' Garber E, Jia H, Zhou JJ, Alba L, Quittell LM, Angst D, Cabana M, Saiman L;CF Infection Control Study Consortium. Infection control knowledge, attitudes, and practices among cystic fibrosis patients and their families. Pediatr Pulmonol.2012 Feb;47(2):144-52.

16. Kidd TJ, Ramsay KA, Hu H, Marks GB, Wainwright CE, Bye PT, Elkins MR, Robinson PJ, Rose BR, Wilson JW, Grimwood K, Bell SC. SharedPseudomonas aeruginosa genotypes are common in Australian cystic fibrosis centres. Eur Respir J 2013; 41:1091-1100.

17. Wiehlmann L, Cramer N, Ulrich J, Hedtfeld S, Weissbrodt H, Tümmler B. Effective prevention of Pseudomonas aeruginosa cross-infection at a cystic fibrosis centre - results of a 10-year prospective study. Int J Med Microbiol. 2012 Mar;302(2):69-77.

\section{Hosted file}

Table Page.docx available at https://authorea.com/users/329806/articles/456717-pseudomonasaeruginosa-among-cystic-fibrosis-patients-epidemiology-risk-factors-and-infectioncontrol 